\title{
Kommentar zu „Gemeinsame Erklärung - Misoprostol für die Behandlung von Blutungen nach der Entbindung in ressourcenarmen Gebieten“
}

\author{
Comment on "Joint Statement - Misoprostol for the Treatment \\ of Postpartum Haemorrhage in Low Resource Settings"
}

Autor

Institut
M. W. Beckmann

Universitätsklinikum Erlangen, Frauenklinik, Erlangen
Die International Confederation of Midwives (ICM) und die International Federation of Gynecology and Obstetrics (FIGO) haben sich in einem gemeinsamen Statement dafür stark gemacht, dass Misoprostol zur Behandlung der postpartalen Hämorrhagie (PPH) eingesetzt werden sollte - wenn Oxytocin nicht verfügbar ist. Dies betrifft vor allem Regionen mit einer eingeschränkten medizinischen Versorgung.

Die PPH ist weltweit die Hauptursache für maternale Morbidität und Mortalität. Dem kann durch das aktive Management - der Gabe von Uterotonika - nach der Geburt des Kindes entgegengewirkt werden. Dennoch ist die postpartale Blutung eine häufige geburtshilfliche Komplikation. Misoprostol hat zahlreiche positive Eigenschaften, die insbesondere in Regionen medizinischer Unterversorgung zum Tragen kommen: es ist günstig, einfach anzuwenden und kann bei Raumtemperatur gelagert werden. In den Fällen, in denen der Goldstandard Oxytocin nicht verfügbar ist, stellt es eine sichere und effektive Option zur Behandlung der PPH dar.
In ihrem Statement empfehlen die ICM und FIGO eine einmalige sublinguale Applikation von $800 \mu \mathrm{g}$. Mehrfache Gaben sollten vermieden und andere Ursachen einer PPH stets in Betracht gezogen werden. Schwere Nebenwirkungen sind selten; zu den häufigeren Nebenwirkungen zählen Fieber, Zittern und gastrointestinale Beschwerden (v.a. Übelkeit und Erbrechen).

Angesichts der verheerenden Folgen einer PPH begrüßen wir die Empfehlung zur Verwendung von Misoprostol. Diese simple Einnahme kann mithilfe weiterer geplanter Maßnahmen (z.B. Training zum Erkennen einer PPH) die Sicherheit der Frauen maßgeblich erhöhen. Auch im Handlungsalgorithmus der PPH-Konsensusgruppe (D-A-CH) ist die Misoprostol-Gabe implementiert. Da wir neben Misoprostol noch auf andere effiziente Medikamente zurückgreifen können, spielt Misoprostol bei uns in der Ersttherapie nur eine untergeordnete Rolle.

Generell gilt jedoch: Eine Empfehlung wie die Gabe von Misoprostol zur Therapie einer postpartalen Hämorrhagie bei Nichtverfügbarkeit von Oxytocin, die Frauen vor schwerer Morbidität und Mortalität schützt, kann nur gutgeheißen werden!
Bibliografie

DOI http://dx.doi.org/

10.1055/s-0034-1368443

Geburtsh Frauenheilk 2014; 74:

429 (c) Georg Thieme Verlag KG

Stuttgart · New York .

ISSN 0016-5751

\section{Korrespondenzadresse}

Prof. Dr. med.

Matthias W. Beckmann

Universitätsklinikum Erlangen

Frauenklinik

Universitätsstr. 21-23

91054 Erlangen

fk-direktion@uk-erlangen.de 